\title{
Calidad de vida en el trabajo: Profesionales de la salud de Clínica Río Blanco y Centro de Especialidades Médicas
}

\section{Quality of life at work: Health Professionals Clinica Rio Blanco and Center Especialidades Médicas}

\author{
Diemen Delgado García ', Miguel Inzulza González², Fabian Delgado García ${ }^{3}$ \\ 1. Unidad Salud Ocupacional, Clínica Río Blanco. Los Andes. Chile. \\ 2. Centro de Especialidades Médicas, Clínica Río Blanco. Los Andes. Chile. \\ 3. Clínica del Trabajador. Manta. Ecuador.
}

Recibido: 09-06-12

Aceptado: 11-07-12

\section{Correspondencia:}

Diemen Delgado García, MD., MPH., Ph.D

Unidad Salud Ocupacional.

Clínica Río Blanco S.A.

Av. Santa María 777

Villa Minera Andina. Los Andes (Chile).

Tfno: (56)034-590547

E-mail: diemen.delgado@clinicarioblanco.cl

Resumen

Objetivo: Identificar un constructo sobre la calidad de vida en el trabajo de los médicos/as y enfermeros/as de Clínica Río Blanco y Centro de Especialidades Médicas.

Métodos y Materiales: Estudio transversal y analítico. Se aplicó un cuestionario de datos socio-laborales integrados por 10 ítems y el instrumento CVT-GOHISALO, el cual consta de 74 ítems y mide la calidad de vida en el trabajo en siete dimensiones.

Resultados: En un grupo de 20 trabajadores entre médicos/as y enfermeros/as, el 40\% cuenta con dos trabajos, el $20 \%$ reporta ausentismo por enfermedad el año previo. El $60 \%$ se desempeñan principalmente en turno mixto. El 40\% tiene una antigüedad de dos a cinco años y el 30\% menos de dos años. El número de horas semanales que dedican al descanso es menor a 20 horas en un 50\% de los trabajadores. Las siete dimensiones evaluadas: soporte institucional para el trabajo, seguridad en el trabajo, integración al puesto de trabajo, satisfacción por el trabajo, bienestar a través del trabajo, desarrollo personal y administración del tiempo libre, reflejan grados de insatisfacción en la calidad de vida en el trabajo.

Conclusiones: Los trabajadores de la salud evaluados muestran una serie de percepciones subjetivas que reflejan una realidad de su medio laboral.

Med Segur Trab (Internet) 2012; 58 (228) 216-223

Palabras clave: Calidad de vida en el trabajo, trabajadores de la salud, grado de satisfacción. 


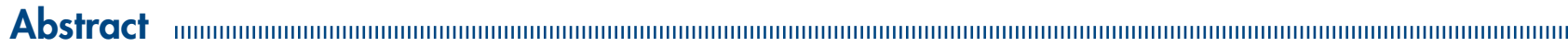

Objective: To identify a construct on quality of life in the work of doctors and nurses Clínica Río Blanco and Center Especialidades Médicas.

Methods and Materials: Cross-sectional and analytical. A questionnaire was social and labor data consisting of 10 question and CVT-GOHISALO instrument, which consists of 74 question and measures the quality of life at work in seven dimensions.

Results: In a group of 20 workers among doctors and nurses, $40 \%$ have two jobs, $20 \%$ reported absenteeism due to illness the previous year. $60 \%$ work mainly in mixed shift. $40 \%$ have a length of two to five years and $30 \%$ less than two years. The number of hours per week devoted to rest is less than 20 hours in $50 \%$ of workers. The seven dimensions assessed: institutional support for work, job security, integration into the workplace, job satisfaction, well-being through work, personal development and leisure management, reflect a degree of dissatisfaction in the quality of work life.

Conclusions: The health workers evaluated show a series of subjective perceptions that reflect a reality of their workplace.

Med Segur Trab (Internet) 2012; 58 (228) 216-223

Key Words: Quality of life at work, health workers, degree of satisfaction. 


\section{INTRODUCCIÓN}

La enfermedad y la atención de ésta constituyen hechos estructurales en toda sociedad; expresan no sólo fenómenos de tipo epidemiológico y clínico sino que también expresan las condiciones sociales, económicas y culturales que toda sociedad inevitablemente procesa a través de sus formas de enfermar, curar y morir ${ }^{1}$.

Desde esta perspectiva, los procesos y relaciones laborales son todavía en la actualidad, parte central de las condiciones sociales que afectan los procesos de salud/ enfermedad, por lo cual la exclusión del trabajo de la descripción y explicación de los procesos personales y colectivos de enfermedad aparece como una clara característica ideológica de la mayoría de la investigación, reflexión e intervención actual sobre la salud de los sujetos y grupos sociales.

Sin embargo, el análisis del saber del trabajador de la salud ha sido reducido al propio saber ${ }^{2}$, escindiéndolo de sus relaciones sociales, fundando dicha escisión en la autonomía científico/técnica de los saberes del médico/a y del enfermero/a.

Para efectos didácticos se hace necesario separar al ser humano ${ }^{3}$, de manera que podamos navegar por las dimensiones que lo constituyen, para conseguir a aprehender y comprender mejor la complejidad de su atención integral en salud en aras de mejorar su calidad de vida.

Nuestra práctica profesional en Clínica Río Blanco y Centro de Especialidades Médicas nos llevo a interrogarnos sobre la calidad de vida en el trabajo de sus profesionales médicos/as y enfermeros/as. Los ejes de nuestra indagación se sostuvieron en las siguientes consideraciones:

1. La índole de la tarea en el campo de la salud posiciona a los trabajadores de la salud en situaciones que implican un contacto permanente con el dolor, la incertidumbre, la angustia, la muerte, así como también con su polo de salud, de vida, de cura ${ }^{4}$.

Estas situaciones estresantes, si bien constituyen el riesgo profesional esperado, en determinadas circunstancias, por factores personales, organizacionales, institucionales o macroestructurales, pierden el valor de cotidianidad profesional sostenible y se convierten en estresores activos, creando vulnerabilidad en los efectores de salud.

2. La complejidad de la producción de conocimientos entre el proceso de trabajo y el proceso de salud / enfermedad / atención constituye un campo complejo, dinámico y multidimensionado que define las prácticas y la calidad de salud 5 .

3. Los actos de salud requieren implicación y el reconocimiento laboral desde su valoración para dimensionarse desde una humanización creativa, responsable y no alienada ${ }^{6}$.

Basándonos en literatura científica que pone de manifiesto el riesgo cardiovascular en trabajadores de la salud ${ }^{7}$, nos condujo a investigar la calidad de vida en el trabajo y a identificar un constructo particular sobre esta problemática en Clínica Río Blanco y Centro de Especialidades Médicas.

\section{MATERIAL Y MÉTODOS}

Se realizó un estudio transversal y analítico en un grupo de 20 trabajadores de la salud en los últimos dos meses del año 2011: 9 médicos/as y 11 enfermeros/as de Clínica Río Blanco y Centro de Especialidades Médicas de la ciudad de Los Andes de la República de Chile. 


\section{Criterios de inclusión}

Médicos/as y enfermeros/as con contrato indefinido en Clínica Río Blanco y Centro de Especialidades Médicas, que cumpla 45 horas semanales asistenciales.

\section{Criterios de exclusión}

1. Trabajadores de la salud con contrato indefinido en Clínica Río Blanco y Centro de Especialidades Médicas, que cumpla 45 horas semanales asistenciales.

2. Médicos/as y enfermeros/as con contrato indefinido en Clínica Río Blanco y Centro de Especialidades Médicas, que cumpla < de 45 horas semanales asistenciales.

3. Médicos/as y enfermeros/as con contrato indefinido en Clínica Río Blanco y Centro de Especialidades Médicas, que cumpla > de 45 horas semanales asistenciales.

\section{Criterios de eliminación}

Médicos/as y enfermeros/as con contrato indefinido en Clínica Río Blanco y Centro de Especialidades Médicas, que cumpla 45 horas semanales asistenciales, que no hayan contestado la encuesta.

\section{Instrumentos}

Un cuestionario de datos socio-laborales integrado por 10 ítems, cuyas variables fueron: sexo, edad, estado civil, grado de estudios, antigüedad, tipo de contratación, turno laboral, otro trabajo, licencias por enfermedad, horas de descanso por semana; y el instrumento para medir la calidad de vida en el trabajo CVT-GOHISALO, evaluando mediante 74 ítems, las siete dimensiones de la calidad de vida ${ }^{8}$.

Se califica utilizando un baremo con puntajes para: alto $(T>60)$ y bajo $(T<40)$ grado de satisfacción en cada una de las dimensiones ${ }^{9}$ : soporte institucional para el trabajo (SIT), seguridad en el trabajo (ST), integración al puesto de trabajo (IPT), satisfacción por el trabajo (SPT), bienestar a través del trabajo (BAT), desarrollo personal (DP) y administración tiempo libre (ATL).

Es un instrumento sencillo, válido y de fácil administración para cualquier trabajador de la salud.

Una vez calificado, se valoran por separado y/o en forma global interpretándose en base al manual para su calificación e interpretación, destacando las dimensiones más sensibles que pueden estar generando una percepción de insatisfacción por parte del trabajador y se puede caracterizar un perfil psicosocial de cada trabajador o del colectivo examinado.

\section{Análisis estadístico}

Se utilizó el modelo estadístico descriptivo en tablas Excel y SPSS 18. Se emplearon los estadísticos paramétricos descriptivos: distribuciones de frecuencia, medidas de tendencia central y variabilidad (media, moda, mediana), rangos mínimos y máximos.

\section{Consideraciones éticas}

Se informó a cada participante seleccionado y voluntario del estudio, el objetivo especifico del mismo. No se realizó ninguna intervención o modificación intencionada en los individuos. Sólo se trabajo en encuesta. Por lo tanto, éste fue un estudio de riesgo mínimo.

No obstante, el autor, se comprometió a manejar la información bajo las normas establecidas por el Código Internacional de Ética Médica ${ }^{10}$. 


\section{RESULTADOS}

Se trata de un grupo laboral en plena edad productiva y madura, $70 \%$ entre 25 y 55 años. El género femenino alcanzo el $50 \%$, la edad promedio fue de 40 años, con un nivel de capacitación universitaria de un $65 \%$ y sólo un $35 \%$ con especialidad; ninguno con grado de maestría. El $40 \%$ cuenta con dos trabajos y el $20 \%$ reporta ausentismo por enfermedad el año previo. El $55 \%$ esta sindicalizado y el $45 \%$ no. El $60 \%$ de los trabajadores se desempeñan principalmente en turno mixto. El $40 \%$ de los trabajadores tiene una antigüedad de dos a cinco años y el $30 \%$ menos de dos años. El número de horas semanales que dedican al descanso es menor a 20 horas en un $50 \%$ de trabajadores. Ver tabla I.

Tabla I. Variables socio laborales médico/as y enfermeros/as de Clínica Río Blanco y * CEM.

\begin{tabular}{|c|c|c|}
\hline Variables significativas & Frecuencia & Porcentaje \\
\hline \multicolumn{3}{|l|}{ Sexo } \\
\hline Masculino & 10 & $50 \%$ \\
\hline Femenino & 10 & $50 \%$ \\
\hline \multicolumn{3}{|l|}{ Estado civil } \\
\hline Soltero & 9 & $45 \%$ \\
\hline Casado & 10 & $50 \%$ \\
\hline Separado & 1 & $5 \%$ \\
\hline \multicolumn{3}{|l|}{ Tipo de contrato } \\
\hline Indefinido sindicalizado & 11 & $55 \%$ \\
\hline Indefinido no sindicalizado & 9 & $45 \%$ \\
\hline \multicolumn{3}{|l|}{ Nivel de estudio } \\
\hline Universitaria & 13 & $65 \%$ \\
\hline Especialidad & 7 & $35 \%$ \\
\hline Maestría & 0 & $0 \%$ \\
\hline \multicolumn{3}{|l|}{ Turno de trabajo } \\
\hline Matutino & 8 & $40 \%$ \\
\hline Mixto & 12 & $60 \%$ \\
\hline \multicolumn{3}{|l|}{ Tiene otro trabajo } \\
\hline $\mathrm{Si}$ & 8 & $40 \%$ \\
\hline No & 12 & $60 \%$ \\
\hline \multicolumn{3}{|l|}{ Ausencia por enfermedad } \\
\hline Si & 4 & $20 \%$ \\
\hline No & 16 & $80 \%$ \\
\hline \multicolumn{3}{|l|}{ Edad } \\
\hline Menores de 24 años & 0 & $0 \%$ \\
\hline Entre 25 - 39 años & 10 & $50 \%$ \\
\hline Entre 40 - 55 años & 4 & $20 \%$ \\
\hline Mayores de 55 años & 6 & $30 \%$ \\
\hline \multicolumn{3}{|c|}{ Horas de descanso por semana } \\
\hline menos de 10 horas & 5 & $25 \%$ \\
\hline menos de 20 horas & 5 & $25 \%$ \\
\hline menos de 30 horas & 4 & $20 \%$ \\
\hline menos de 40 horas & 4 & $20 \%$ \\
\hline menos de 50 horas & 2 & $10 \%$ \\
\hline
\end{tabular}




\begin{tabular}{lcc}
\hline \multicolumn{1}{c}{ Variables significativas } & Frecuencia & Porcentaje \\
\hline $\begin{array}{l}\text { Antigüedad en la empresa } \\
\text { < } 2 \text { años }\end{array}$ & 6 & $30 \%$ \\
2 a 5 años & 8 & $40 \%$ \\
6 a 10 años & 1 & $5 \%$ \\
11 a 15 años & 1 & $5 \%$ \\
16 a 20 años & 0 & $0 \%$ \\
más de 20 años & 4 & $20 \%$ \\
\hline \multicolumn{3}{c}{ Fuente: Directa } \\
\\
\multirow{2}{c}{ CEM (Centro Médico de Especialidades) }
\end{tabular}

Respecto a la calidad de vida en el trabajo, calificado de acuerdo al instrumento CTVGOHISALO, se obtuvieron las siguientes interpretaciones por cada dimensión. Ver Tabla II.

Tabla II. CVT-GOHISALO aplicado a médico/as y enfermeros/as de Clínica Río Blanco y * CEM.

\begin{tabular}{lcrrrrrrr}
\hline \multicolumn{1}{c}{ Grado de satisfacción } & Puntuación T & SIT & ST & IPT & SPT & BAT & DP & ATL \\
\hline Satisfacción alta & $>60-99$ & 6 & 4 & 2 & 2 & 1 & 1 & 0 \\
Satisfacción baja & $<40$ & 14 & 16 & 18 & 18 & 19 & 19 & 20 \\
\hline \multicolumn{8}{c}{ Fuente: Directa } \\
& * CEM (Centro Médico de Especialidades)
\end{tabular}

\section{Dimensión soporte institucional para el trabajo (SIT)}

Catorce trabajadores obtuvieron puntuación por debajo de T-40 en esta dimensión. Son personas que reportan insatisfacción en los procesos del trabajo, dificultades en la realización de las tareas y por ende, baja motivación en las funciones que le son asignadas. No visualizan oportunidades de progreso en el puesto o función que desempeñan y sienten insatisfacción personal, con limitada oportunidad de expresar su opinión por temor a represalias.

Seis trabajadores obtuvieron puntuación por encima de T-60 en esta dimensión. Se consideran personas con actividades o tareas claras y acordes con su motivación, perfil profesional o puesto laboral. Sienten que su actividad laboral les permite alcanzar promociones dentro de la institución o empresa.

\section{Dimensión seguridad en el trabajo (ST)}

16 trabajadores obtuvieron puntuación por debajo de T-40 en esta dimensión. Por lo general, son personas con poca satisfacción en su trabajo. Perciben una baja remuneración por el trabajo realizado. Sienten injusticias en las evaluaciones. Es frecuente que se reporten con problemas de salud relacionados a las condiciones de trabajo.

Cuatro trabajadores obtuvieron puntuación por encima de T-60 en esta dimensión. Sienten que su trabajo les ofrece los medios económicos y sociales para cubrir sus necesidades y las de sus seres queridos. Se describen con buena capacitación para seguir en el puesto o tener una carrera institucional.

\section{Dimensión integración al puesto de trabajo (IPT)}

Dieciocho trabajadores obtuvieron puntuación por debajo de T-40 en esta dimensión. Suelen expresar inconformidad con el cumplimiento de los objetivos institucionales, se desmotivan con facilidad y responsabilizan a los demás de los fracasos laborales.

Dos trabajadores obtuvieron puntuación por encima de T-60 en esta dimensión. Están motivados para superar retos y muy dispuestos a ser líderes. Tienen capacidad de 
crear ambientes de trabajo satisfactorios para los demás, encontrar alternativas para motivar a sus compañeros o estar dispuestos a cooperar como subordinados.

\section{Dimensión satisfacción por el trabajo (SPT)}

Dieciocho trabajadores obtuvieron puntuación por debajo de T-40 en esta dimensión. Se sienten insatisfechos por su actividad. Suelen tener otras funciones fuera de la institución o empresa, su dedicación por lo tanto es parcial.

Dos trabajadores obtuvieron puntuación por encima de T-60 en esta dimensión. Suelen ser comprometidos con la misión de la institución. Son personas que muestran los aspectos positivos del trabajo, se autovaloran con orgullo por pertenecer a la institución y sienten satisfacción por la retribución que se ofrece a sus funciones.

\section{Dimensión bienestar logrado a través del trabajo (BAT)}

Diecinueve trabajadores obtuvieron puntuación por debajo de T-40 en esta dimensión. Los trabajadores perciben riesgo en la salud física o emocional. Se sienten insatisfechos con la remuneración adquirida por su trabajo y por consecuencia, responsabilizan el entorno laboral por su precariedad.

Un trabajador obtuvo puntuación por encima de T-60 en esta dimensión. Suele estar satisfecho con el progreso personal, social y económico que han obtenido por su trabajo.

\section{Dimensión desarrollo personal (DP)}

Diecinueve trabajadores obtuvieron puntuación por debajo de T-40 en esta dimensión. Los trabajadores expresan inseguridad por los logros alcanzados, se sienten vulnerables ante el trato con los usuarios o compañeros de trabajo. Pueden considerar que el trabajo le ha disminuido su capacidad física o emocional.

Un trabajador obtuvo puntuación por encima de T-60 en esta dimensión. Tiene buen estado de ánimo, es optimista y de trato amable. Trasmite a los demás su entusiasmo, alegría y bienestar.

\section{Dimensión administración de tiempo libre (ATL)}

Veinte trabajadores obtuvieron puntuación por debajo de T-40 en esta dimensión. Prolongan su jornada laboral o suelen llevar trabajo a casa, lo que repercute en su descanso o recreación. Por lo común, no registran sus actividades y el tiempo que les lleva. Su laxitud puede ponerle en riesgo nutricional o emocional, es decir, incorporar algunas adicciones o tener conflictos interpersonales, dado el incumplimiento de la actividad recreativa y social.

\section{DISCUSIÓN}

Este estudio constituye el primer intento para diagnosticar o conocer el grado de satisfacción con la calidad de vida en el trabajo percibida por trabajadores de la salud en Chile, teniendo como antecedente solamente el estudio de validación del instrumento aplicado para medir el constructo ${ }^{11}$. De aquí la importancia, ya que podrá ser utilizado como referencia en otras instituciones de salud pública y clínicas privadas donde estos profesionales se desempeñan.

La satisfacción en todas las dimensiones evaluadas con el instrumento CVTGOHISALO con puntuación por debajo de T-40 es elevada en esta muestra seleccionada. Ver tabla II.

Se concluye que los trabajadores de la salud evaluados muestran una serie de percepciones subjetivas que reflejan una realidad ${ }^{3}$ de su medio laboral.

Representan además una actitud subyacente que podría afectar los objetivos de la institución y la calidad de sus servicios ofrecidos, así como la posibilidad de manifestaciones 
de inconformidad en el futuro, a menos que se detecten las causas de la insatisfacción, sean analizadas y satisfechas gradualmente.

Se sugiere diseñar estrategias o programas de intervención que puedan ofrecer una ruta para acceder a una mayor satisfacción profesional y una mejor calidad de vida en el trabajo.

\section{AGRADECIMIENTOS}

Al Dr. Miguel Ángel Inzulza González Director Médico de Clínica Río Blanco y del Centro de Especialidades Medicas, por su innegable apoyo en el desarrollo de esta investigación. Y a mis compañeros de trabajo que participaron en este estudio que indudablemente abrirá espacios al conocimiento de la calidad de vida en el trabajo en la dimensión cualitativa del ser.

\section{REFERENCIAS BIBLIOGRÁFICAS}

1. Timio M. Clases sociales y enfermedad. Introducción a una epidemiologia diferencial. México: Editorial Nueva Imagen; 1978.

2. Menéndez E. El modelo médico y la salud de los trabajadores. Salud Colectiva 2005;1(1):9-32.

3. Merleau-Ponty M. Fenomenología de la percepción. Barcelona: Península; 1975.

4. Maslach C and Jackson SE. Burnout in the professions. Citado en Moreno-Jiménez, et al. Op. Cit., 1982.

5. Zaldúa G, Lodieu MT, Koloditzky D. La salud de los trabajadores de la salud. Estudio del síndrome de Burnout en hospitales públicos de la ciudad autónoma de Buenos Aires. Revista del Hospital Materno Infantil Ramón Sarda 2000;19(4):167-72.

6. Breilh J. La epidemiologia en la humanización de la vida. Convergencias y desencuentros de las corrientes. Bahía; 1995

7. Robaina C, Martínez R, Robaina F y Plunket D .Riesgo cardiovascular en trabajadores de la salud. Rev Cubana Med Gen Integr 1999;15(2):115-22.

8. González R, Hidalgo G, Salazar JG, Preciado ML. Instrumento para medir la calidad de vida en el trabajo (CVT-GOHISALO): manual para su aplicación y su interpretación. Guadalajara, México: Ed. de la Noche. 2009.

9. Salazar JG, Guerrero JC. Calidad de vida del trabajador. Colombia: Universidad Libre de Cali. 2009.

10. Klimovsky E, Saidon P, Nudelman L, Bignone I. Declaración de Helsinki. Sus vicisitudes en los últimos cinco años. Medicina (Buenos Aires) 2002; 62:365-70.

11. González R. Calidad de vida en el trabajo: elaboración y validación de un instrumento en prestadores de servicios de salud [tesis]. México: Universidad de Guadalajara. 2007.

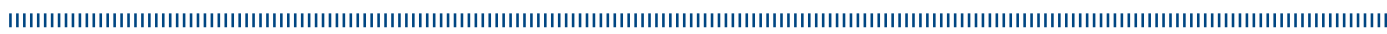

